\title{
El trabajo nocturno podría asociarse a mayor incidencia de enfermedad coronaria
}

Night shift work may be associated with a greater incidence of coronary disease

Vetter C, y col. JAMA. 2016;315(16):1726-34.

\section{Objetivos}

Determinar la asociación entre el trabajo nocturno y la aparición de enfermedad coronaria.

\section{Diseño, lugar y pacientes}

Cohorte prospectiva que incluyó 189.158 enfermeras (pertenecientes a la cohorte de los Estudios de la Salud de las Enfermeras, conocido en inglés como Nurses' Health Studies), inicialmente libres de enfermedad coronaria, con un seguimiento de 24 años. Este estudio, realizado en los EE.UU., incluyó dos poblaciones: NHS (periodo 1988 a 2012, n=73.623) y NHS2 (periodo 1989 a 2013, n=115.535).

\section{Evaluación de los factores de riesgo}

Se evaluó la rotación en turno nocturno (definido como tres o más noches en el turno nocturno por mes) en distintos periodos del estudio. También se midieron covariables relacionadas con la historia

Tabla 1. Asociación entre los años de trabajo nocturno y la incidencia de eventos cardiovasculares

\begin{tabular}{|c|c|c|c|c|c|}
\hline \multirow[b]{2}{*}{ Estudio } & \multicolumn{4}{|c|}{ Categorías de años de trabajo nocturno } & \multirow[b]{2}{*}{$\begin{array}{c}\text { Valor de p } \\
\text { para } \\
\text { tendencia }\end{array}$} \\
\hline & $\begin{array}{c}\text { Ninguno } \\
\text { HR (IC 95\%) } \\
\text { Incidenciaa }^{\text {a }}\end{array}$ & $\begin{array}{c}0 \text { a } 5 \\
\text { HR (IC 95\%) } \\
\text { Incidencia }^{\mathrm{a}}\end{array}$ & $\begin{array}{c}5 \text { a } 9 \\
\text { HR (IC 95\%) } \\
\text { Incidencia }\end{array}$ & $\begin{array}{l}\text { Más de } 10 \\
\text { HR (IC 95\%) } \\
\text { Incidencia }\end{array}$ & \\
\hline NHS primera mitad del seguimiento & 1 [Referencia] 367.3 & $1,1(1,01$ a 1,21$) 382.4$ & $1,19(1,03$ a 1,39$) 483.1$ & $1,27(1,13$ a 1,42$) 494.4$ & $<0,001$ \\
\hline NHS segunda mitad del seguimiento & 1 [Referencia] 436.6 & $0.98(0.92$ a 1.05$) 424.8$ & $1.08(0.96$ a 1.21$) 520.7$ & $1.13(1.0 \mathrm{a} 1.24) 556.2$ & $<0,004$ \\
\hline NHS 2 & 1 [Referencia] 122.6 & $1.05(0.97$ a 1.13$) 130.6$ & $1.12(0.99 \mathrm{a} 1.26) 151.6$ & $1.15(1.01 \mathrm{a} 1.32) 178$ & $<0,01$ \\
\hline
\end{tabular}

HR: Hazard ratio. IC 95\%: intervalo de confianza del 95\%. a Tasas ajustadas por edad por cada 100.000 personas-año.

En el NHS, la asociación entre la duración del trabajo nocturno y la enfermedad fue más fuerte en la primera mitad del seguimiento que en la segunda mitad ( $p=0,02$ para la interacción), lo que sugiere un menor riesgo después del cese del trabajo por turnos nocturnos. El mayor tiempo transcurrido desde que se abandonó el trabajo por turnos se asoció con una disminución del riesgo de enfermedad coronaria en el NHS2.

\section{Conclusiones}

Entre las mujeres que trabajaban como enfermeras registradas, la clínica, datos antropométricos, dieta y estilo de vida con actualización bienal.

\section{Medición de resultados principales}

Se evaluó la presencia de enfermedad coronaria por el reporte de los participantes y el análisis independiente de las historias clínicas. Se incluyeron como desenlaces la enfermedad coronaria (cirugía de revascularización miocárdica, angina pectoris, angioplastia y colocación de stents), el infarto de miocardio fatal y la muerte de causa cardiovascular.

\section{Resultados principales}

Durante el seguimiento, ocurrieron 7.303 casos de enfermedad coronaria incidente en el grupo NHS (edad media al inicio del estudio: 54,5 años) y 3.519 en el grupo NHS2 (edad media al inicio del estudio: 34,8 años). Se realizaron modelos de ajuste multivariado de riesgos proporcionales de Cox (ver tabla 1).

\section{Comentario}

El estudio muestra que la mayor exposición al trabajo en turnos nocturnos en enfermeras se asoció a un aumento pequeño, aunque estadísticamente significativo, de cardiopatía coronaria. Estos resultados sugieren que el riesgo podría reducirse si se disminuye el trabajo nocturno. Desde hace tiempo se conoce la influencia de nuestro reloj biológico en los procesos metabólicos. Los ritmos circadianos existen en todos los seres vivos, desde los organismos unicelulares hasta los seres humanos, permitiendo su adaptación interna al ciclo de luz/oscuridad ambiental. La desincronización de estos ritmos de aproximadamente 24 horas de duración puede tener efectos adversos en la salud. Un metaanálisis realizado en 2012 mostró un aumento del riesgo cardiovascular, de infartos, y en menor medida, de isquemia cerebral ${ }^{1}$. De igual manera, se evidenció un incremento de calcifi- mayor duración del turno rotatorio nocturno se asoció con un aumento absoluto estadísticamente significativo pero pequeño en el riesgo de desarrollar enfermedad coronaria. Se necesita más investigación para explorar si la asociación está relacionada con horas de trabajo específicas y características individuales.

Fuente de financiamiento: Centro de Control y Prevención de Enfermedades; Instituto Nacional de Salud y Seguridad Ocupacional; Fundación de Investigación Alemana.

Ana María Mosca [ Departamento de Enfermería Clínica. Instituto Universitario Hospital Italiano de Buenos Aires. ana.mosca@hospitalitaliano.org.ar]

Mosca AM. El trabajo nocturno podría asociarse a mayor incidencia de enfermedad coronaria. Evid Act Pract Ambul 2018;20(2):61. Comentado de: Vetter C, y col. Association Between Rotating Night Shift Work and Risk of Coronary Heart Disease Among Women. JAMA. 2016;315(16):172634. PMID: 27115377 